\title{
Mouse cytosolic acetyl-CoA hydrolase, a novel candidate for a key enzyme involved in fat metabolism: cDNA cloning, sequenc- ing and functional expression ${ }^{* *}$
}

\author{
Naoya Suematsu, Kazuki Okamoto and Fumihide Isohashi ${ }^{\varpi}$ \\ Department of Biochemistry, St. Marianna University School of Medicine, Kanagawa, Japan
}

Received: 30 April, 2002; revised: 25 September, 2002; accepted: 03 October, 2002

Key words: acetyl-CoA hydrolase, PCR, cDNA sequence, Spodoptera frugiperda, functional expression

A cytosolic acetyl-CoA hydrolase (CACH) cDNA has been isolated from mouse liver cDNA library and sequenced. Recombinant expression of the cDNA in insect cells resulted in overproduction of active acetyl-CoA hydrolyzing enzyme protein. The mouse CACH cDNA encoded a 556-amino-acid sequence that was $93.5 \%$ identical to rat CACH, suggesting a conserved role for this enzyme in the mammalian liver. Database searching shows no homology to other known proteins, but reveals homological cDNA sequences showing two single-nucleotide polymorphisms (SNPs) in the CACH coding region. The discovery of mouse CACH cDNA is an important step towards genetic studies on the functional analysis of this enzyme by gene-knockout and transgenic approaches.

An extramitochondrial (cytosolic) acetylCoA hydrolase $(\mathrm{CACH})$, which hydrolyzes acetyl-CoA to acetate and CoA, has been first detected in rat liver and kidney ( $5 \%$ of the activity in liver cytosol) (Prass et al., 1980). The enzyme activity greatly increases in liver both in opposite metabolic states: in enhanced fatty acid oxidation and in heightened fatty acid synthesis (Matsunaga et al., 1985). Marked induction in liver was also observed

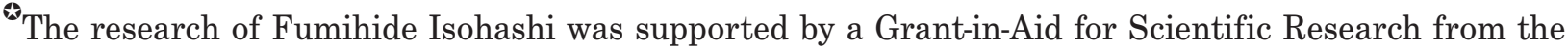
Ministry of Education, Science, Sports and Culture of Japan and by a grant from the Vitamin Society of Japan.

${ }^{凶}$ Author for correspondence: Fumihide Isohashi, Department of Biochemistry, St. Marianna University School of Medicine, Sugao, Miyamae-ku, Kawasaki, Kanagawa 216-8511, Japan; tel. (81 44) 977 8111; fax: (81 44) 976 7553; e-mail: n2sue@marianna-u.ac.jp

Abbreviations: $\mathrm{CACH}$, cytosolic acetyl-CoA hydrolase; EST, expressed sequence tag; MOI, multiplicity of infection; SNP, single-nucleotide polymorphism.

* Nucleotide sequence data are available in the DDBJ/EMBL/GenBank databases under the accession number AB078618.

Acetyl-CoA hydrolase (EC 3.1.2.1); acyl-CoA thioesterase (EC 3.1.2.2).
} 
by 2-( $p$-chlorophenoxy)isobutyric acid (Ebisuno et al., 1988), a hypolipidemic drug or peroxisome proliferator, which enhances rat liver mitochondrial and peroxisomal $\beta$-oxidation (Mannaerts et al., 1979) and increases cytosolic CoA level (Horie et al., 1986; Berge et $a l ., 1983)$. These findings suggested some specific role of the enzyme in fat metabolism of the tissue and its physiological role has been postulated to be to supply cytosolic free CoA for both fatty acid synthesis and oxidation (Matsunaga et al., 1985).

The enzyme had escaped previous purification due to its extreme cold lability (Isohashi et al., 1983a; Suematsu et al., 1996) and a lack of activity in the absence of ATP (Söling \& Rescher, 1985; Nakanishi et al., 1988). We successfully purified this enzyme at room temperature (Ebisuno et al., 1989; Nakanishi et al., 1993) and demonstrated that at room temperature it is usually present as active forms: homodimer (135 $\mathrm{kDa})$ and homotetramer $(240 \mathrm{kDa})$ whose $K_{\mathrm{m}}$ values for acetyl-CoA are $170 \mu \mathrm{M}$ and $60 \mu \mathrm{M}$, respectively (Isohashi et al., 1983b). But at $4^{\circ} \mathrm{C}$, they dissociate into an inactive monomer (63 kDa) (Isohashi et al., 1984). Our in vitro study further demonstrated that $\mathrm{CACH}$ is an allosteric enzyme regulated by ATP (activator) and ADP (inhibitor) (Isohashi et al., 1983a), suggesting that it is presumably a key enzyme. It should be noted that ATP is not a substrate, and that its effect is due to allosteric interaction. The effect of ATP did not require the presence of $\mathrm{Mg}^{2+}$ and the production of neither ADP nor inorganic phosphate was detected (Prass et al., 1980).

We cloned rat $\mathrm{CACH}$ in a previous study (Suematsu et al., 2001), demonstrating that it is a novel thioesterase. Here we report molecular cloning and recombinant expression of a mouse homologue cDNA, as the second example after that of the rat. The cloning of the mouse enzyme allows genetic studies using mouse models such as gene-knockout and transgenic mice to determine the physiological functions of this enzyme.

\section{MATERIALS AND METHODS}

Acetyl-CoA was synthesized as described previously (Simon \& Shemin, 1953). All other chemicals and reagents used were of analytical grade, or better.

Enzyme assay. Enzyme activity was routinely assayed at $25^{\circ} \mathrm{C}$ as previously described (Prass et al., 1980). One unit of the enzyme is that required to hydrolyze $1 \mu \mathrm{mol}$ of acetyl$\mathrm{CoA} \times \min ^{-1}$ under conditions of the assay. Acetyl-CoA hydrolase activity was estimated by subtracting the nonenzymatic rate measured in $2 \mathrm{mM}$ ADP, which inhibits the enzymatic activity, from that observed in $2 \mathrm{mM}$ ATP. All determinations were carried out in triplicate.

cDNA cloning from mouse liver cDNA library. A mouse liver cDNA library $\left(1 \times 10^{7}\right.$ independent clones, SuperScript cDNA library, Life Technologies, Inc., Tokyo, Japan) derived from mRNA from 8-week old, male C57BL/6J mice was used as a template to amplify mouse CACH cDNA. The target cDNA was amplified by PCR with Platinum Pfx DNA Polymerase (Life Technologies, Inc.), using synthesized oligonucleotide primer set of S1/A1 (Table 1), and sequenced. The PCR product was subjected to an additional nested PCR step, using an anchor primer set of S2/A2 (Table 1), whose 5 '-sites included additional restriction sites to facilitate subsequent cloning. The amplified cDNA was inserted into the SacI/BglII site of the baculovirus transfer vector pTriEx-4. The resulting recombinant transfer vector was designated pTriEx-4/cach (Fig. 1A). The expressed recombinant protein will be a $6 x H i s-f u s i o n$ protein. This feature allows affinity purification on $\mathrm{Ni}^{2+}$-charged resin, which may greatly simplify isolation of the His-tagged enzyme protein.

Bioinformatics. Nucleotide and protein sequence homology was searched with a computer using BLAST 2.0, and sequence alignments were made using the DiAlign computer algorithm (Genomatix Software GmbH, München, Germany). 
Table 1. Oligonucleotide primers used.

Nucleotide positions are numbered as in Fig. 2. ATG start codon and TAA stop codon are highlighted in bold type. S, sense; A, antisense; CDS, coding sequence.

\begin{tabular}{llcccc}
\hline Primer & Sequence $\left(5^{\prime}-3^{\prime}\right)$ & Length $(\mathrm{mer})$ & $t_{\mathrm{m}}\left({ }^{\circ} \mathrm{C}\right)$ & Position & Location \\
\hline S0 & TTTGGCAAAGAATTGGATCGGACC & 24 & 58.8 & (specific to pTriEx-4 vector) \\
S1 & CAGGACGATGGAGTCGATGG & 20 & 60.4 & $-7 / 13$ & 5 'terminus \\
S2 & ATGAGCTCATGGAGTCGATGGTGGC & 25 & 63.8 & $1 / 17$ & anchor primer \\
S3 & TTCACTTAAAACCTGTCCTGCTTC & 24 & 57.1 & $369 / 392$ & CDS \\
S4 & TTTACAATGCTGTTGATGACCAGG & 24 & 57.1 & $867 / 890$ & CDS \\
S5 & GACTTTGTGGTGCTTGTGTCACG & 23 & 60.5 & $1355 / 1377$ & CDS \\
A0 & TCGATCTCAGTGGTATTTGTGAGC & 24 & 58.8 & (specific to pTriEx-4 vector) \\
A1 & GAGTTGACCATTTATAACACACTTTTAAGTCC & 32 & 59.3 & $1686 / 1655$ & 3 '-terminus \\
A2 & GCAGATCTTATAACACACTTTTAAGTCCATCAGG & 34 & 61.8 & $1675 / 1649$ & anchor primer \\
A3 & AGCAGGACAGGTTTTAAGTGAACC & 24 & 58.8 & $390 / 367$ & CDS \\
A4 & GAGTTTCTCCTGGTCATCAACAGC & 24 & 60.5 & $898 / 875$ & CDS \\
A5 & GTGACACAAGCACCACAAAGTCC & 23 & 60.5 & $1376 / 1354$ & CDS \\
\hline
\end{tabular}

Recombinant expression. The recombinant transfer vector pTriEx-4/cach (Fig. 1A) was cotransfected with BaculoGold viral DNA into monolayer Spodoptera frugiperda (Sf9) insect cells in an optimized TNM-FH Insect Medium according to the procedures provided with the expression system (BD PharMingen). The recombinant virus was amplified, isolated, partially sequenced and subsequently used to transform $S f 9$ cells for recombinant expression of the His-tagged enzyme protein (Fig. 1B) according to the man-

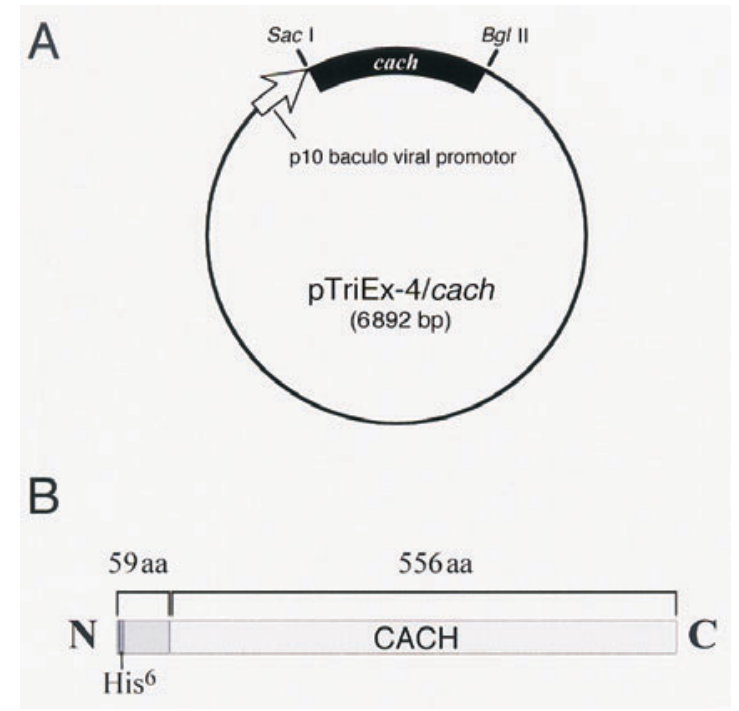

ufacturer's instructions. To assess the recombinant expression, infected cells were pelleted, rinsed twice in phosphate-buffered saline (PBS) and then lysed in a minimal volume (1.4 mL for $2 \times 10^{7}$ cells) of buffer A containing $25 \mathrm{mM}$ Tris/phosphate (pH 7.8), $2 \mathrm{mM}$ dithiothreitol (DTT), $2 \mathrm{mM}$ 1,2-diaminocyclohexane- $N, N, N^{\prime}, N^{\prime}$-tetraacetic acid, $10 \%$ glycerol and 1\% Triton X-100. The cell lysate was clarified by centrifugation at $15000 \times \boldsymbol{g}$ for $10 \mathrm{~min}$ at $25^{\circ} \mathrm{C}$ and then subjected to the enzyme assay.

Figure 1. Schematic depiction of strategy for recombinant expression of a full-length cDNA of mouse cytosolic acetyl-CoA hydrolase.

A. Construction of a recombinant baculovirus transfer vector containing the complete coding region of the mouse cytosolic acetyl-CoA hydrolase. The full-length cDNA encoding mouse $\mathrm{CACH}$ was inserted into the multiple cloning site of the baculovirus transfer vector pTriEx-4 at the SacI/BglII site. The resultant vector was 6892-bp long and designated pTriEx-4/cach. B. Schematic representation of the recombinant $\mathrm{CACH}$ protein containing a polyhistidine $(6 \mathrm{xHis}) \mathrm{tag}$. 


\section{RESULTS}

Characterization of the full-length $\mathrm{CACH}$ cDNA. As outlined in Fig. 2, the CACH cDNA obtained from C57BL/6J mice is 1689-nucleo- tides long and comprises a coding region with an ORF of $1668 \mathrm{bp}$, which starts with an ATG initiation codon (shown in bold face), whose first nucleotide is numbered as +1 , and terminates with a TAA stop codon (positions

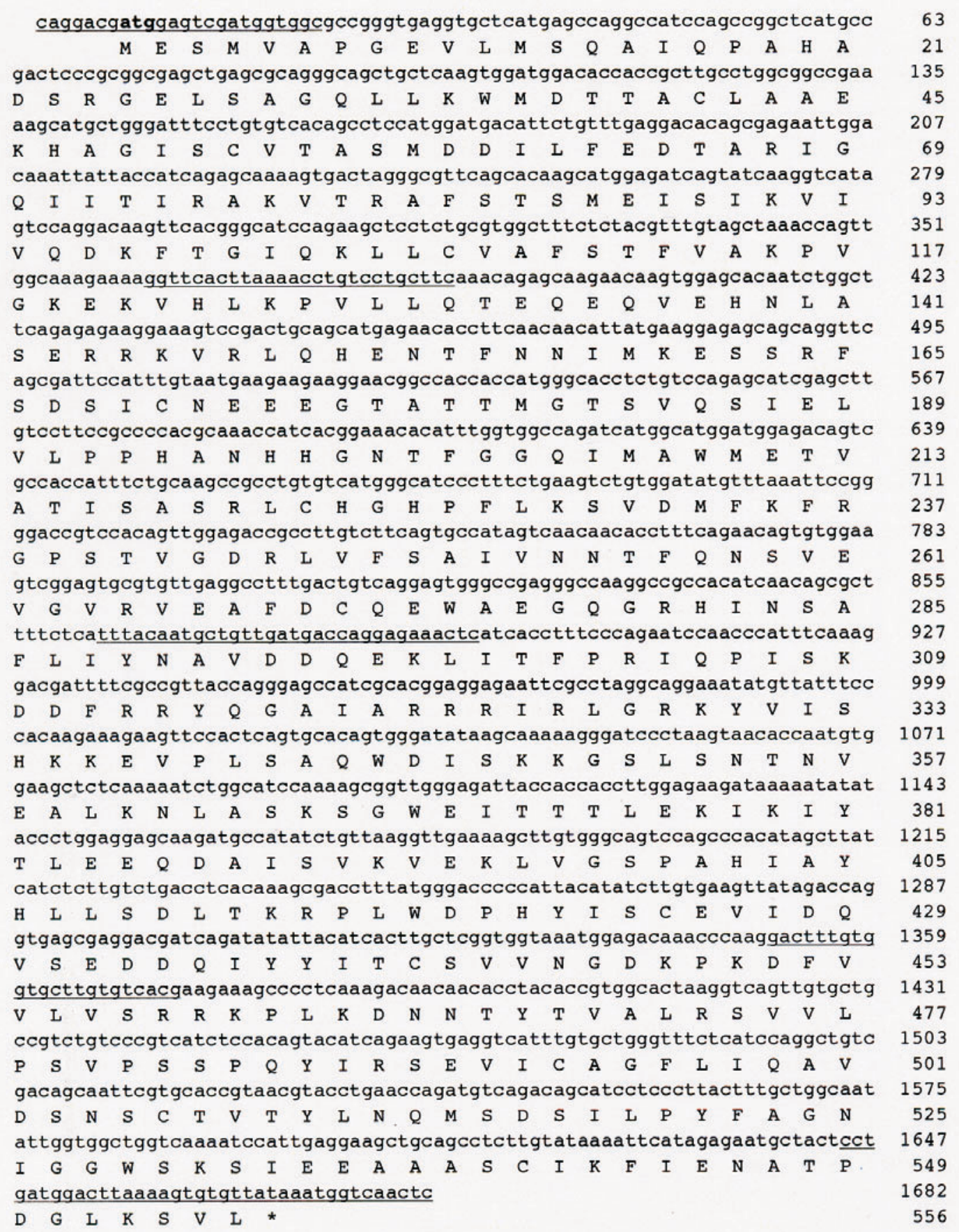

Figure 2. Nucleotide sequence of the full-length cDNA encoding mouse cytosolic acetyl-CoA hydrolase and its deduced amino-acid sequence.

The nucleotide and predicted amino-acid residues are numbered on the right from the first base of the ATG start codon (shown in bold face). The underlined nucleotide sequences correspond to the gene-specific primers used for sequencing (Table 1). The asterisk denotes the TAA stop codon. Sequence data have been submitted to DDBJ under the accession number AB078618. 
Table 2. Comparison of the nucleotide and deduced amino-acid sequence of mouse cytosolic acetyl-CoA hydrolase with databases.

Percentage identities of nucleotide and amino-acid residues are shown.

\begin{tabular}{llllllll}
\hline & $\begin{array}{l}\text { GenBank ac- } \\
\text { cession no. }\end{array}$ & Length & Residues identical with mouse CACH \\
\hline $\begin{array}{l}\text { Mouse cytosolic acetyl-CoA } \\
\text { hydrolase }\end{array}$ & AB078618 & 556 aa & & & & & \\
Mouse cDNA to mRNA & AK004905 & 556 aa & $1667 / 1668$ & bp & $(99.9 \%)$, & $556 / 556$ aa & $(100 \%)$ \\
Mouse expressed sequence tag 1 & AI425375 & 189 aa & $566 / 567$ & bp & $(99.8 \%)$, & $188 / 189$ aa (99.5\%) \\
Mouse expressed sequence tag 2 & AA066584 & 114 aa & $343 / 343$ & bp & $(100 \%)$, & $114 / 114$ aa (100\%) \\
Rat cytosolic acetyl-CoA hydrolase & AB040609 & 556 aa & $1540 / 1668$ & bp & $(92.2 \%)$, & $520 / 556$ aa (93.5\%) \\
\hline
\end{tabular}

1669-1671, shown with an asterisk). The first AUG should be the initiator (Fig. 2) because it agrees well with Kozak's rule (Kozak, 1987; 1991). The cDNA encoded a protein of 556 amino-acid residues with calculated molecular mass of $61761 \mathrm{Da}$ and its sequence was $93.5 \%$ identical to that of the rat homologue (Table 2, referred to as rCACH in Fig. 3). PSORT WWW Server (http://psort.nibb.ac.jp) predicted cytoplasmic localization of the $\mathrm{CACH}$ protein from the cDNA sequence not containing any targeting signals for organella includ-

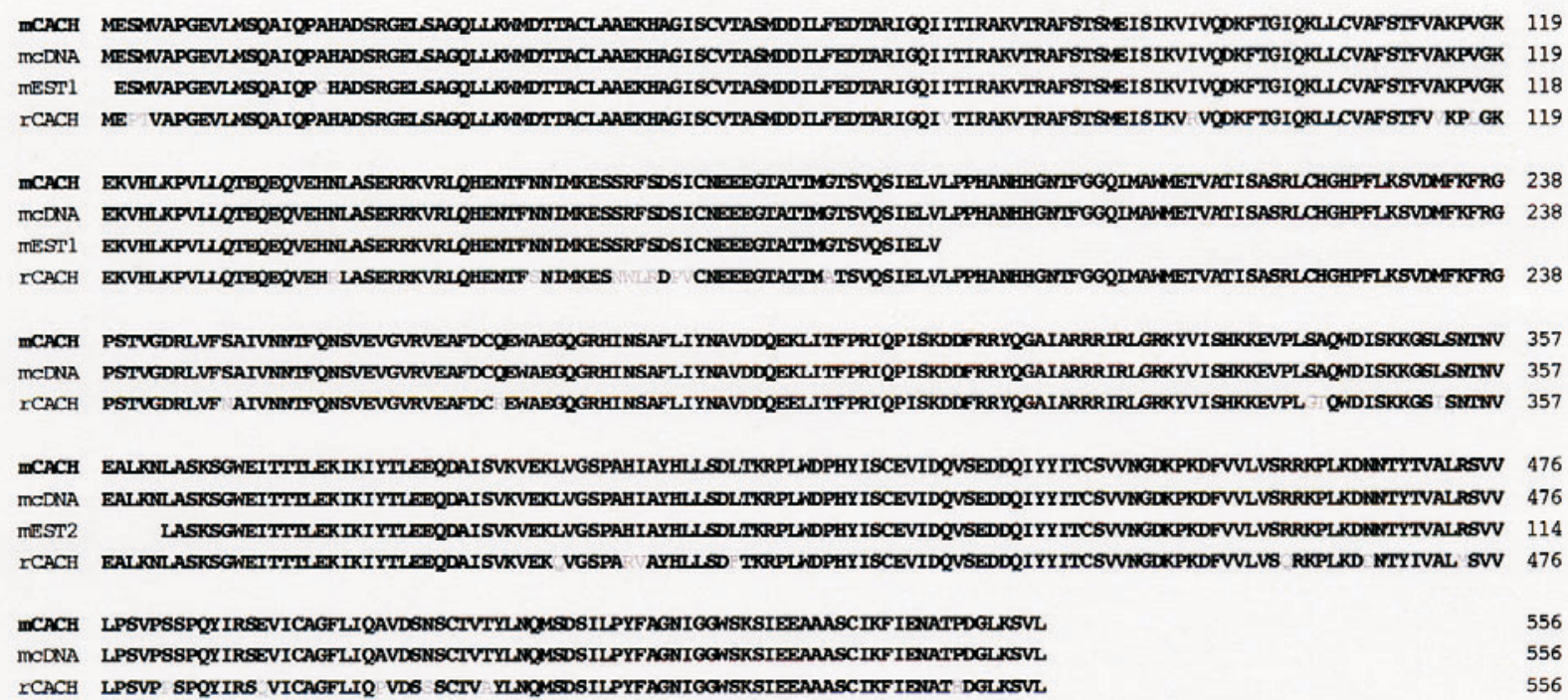

Figure 3. Comparison of the deduced amino-acid sequence of mouse cytosolic acetyl-CoA hydrolase with protein sequence databases.

Amino-acid sequences were recovered from the DDBJ/EMBL/GenBank databases and aligned using the DiAlign algorithm (Genomatix Software GmbH, München, Germany). The amino-acid residues are numbered on the right. Identical amino acids conserved between at least two of the sequences are shown highlighted in bold face. The sequences compared are as follows: mCACH, mouse cytosolic acetyl-CoA hydrolase (this study, GenBank accession number AB078618); mcDNA, C57BL/6J mouse Mus musculus full-length cDNA to mRNA related to acyl-CoA thioester hydrolase family protein (AK004905); mEST1, Soares mouse NML Mus musculus EST clone (AI425375); mEST2, Stratagene mouse kidney (\#937315) Mus musculus EST clone (AA066584); rCACH, rat cytosolic acetyl-CoA hydrolase (AB040609). 
ing peroxisome, mitochondria and nucleus. The sequence is available from DDBJ/ EMBL/GenBank under the accession number AB078618. A partially deleted $\mathrm{CACH}$ cDNA was also isolated: it spanned $1586 \mathrm{bp}$, lacking 103 bp (positions 1420-1522) followed by a TAA termination codon, and contained a shorter ORF encoding only the N-terminal 473 amino-acid residues with calculated molecular mass of $52978 \mathrm{Da}$ (data not shown).

Homology search. Database searches revealed sequence similarity only to hypothetical proteins of unestablished function. A cDNA to mouse liver mRNA, related to an acyl-CoA thioester hydrolase family protein (GenBank accession number AK004905, referred to mcDNA in Fig. 3), was found to encode the same protein (Table 2), although containing a single C-to-T transition at nucleotide position 418. Further, an expressed sequence tag (EST) clone (567 bp) derived from mouse liver (AI425375, referred to as mouse expressed sequence tag 1 in Table 2 and mEST1 in Fig. 3) corresponded to the mouse $\mathrm{CACH}$ cDNA at nucleotide positions 4-570 with a

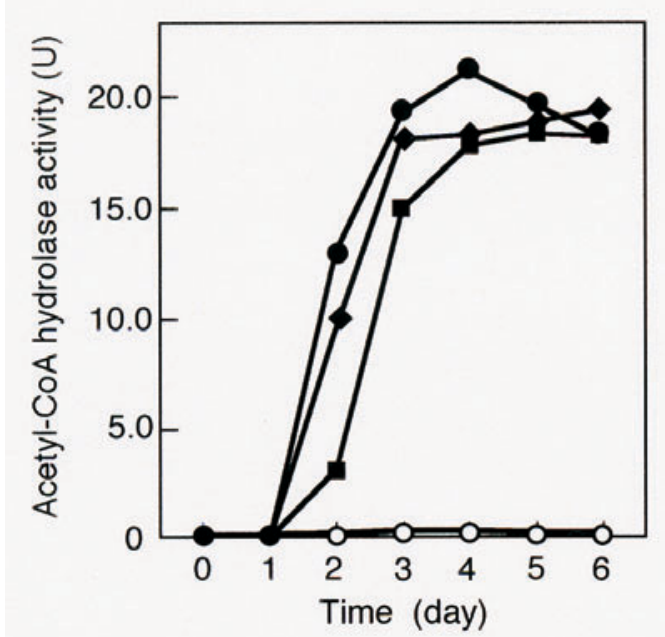

Figure 4. Recombinant expression of mouse cytosolic acetyl-CoA hydrolase cDNA in Spodoptera frugiperda (Sf9) insect cells.

A representative time-course expression pattern (0, 1 , $2,3,4,5,6$ days) of recombinant acetyl-CoA hydrolase is shown. Cell lysates from Sf9 cells infected at multiplicity of infection (MOI) $1(\square), 5(\diamond)$ and $10(\mathbf{O})$ or uninfected $(O)\left(29 \times 10^{5}\right.$ cells at day 0$)$ on a 12 -well plate were prepared and analyzed for the enzyme activity. single C-to-G substitution at nucleotide position 56, causing a missense mutation A19G, suggesting an allele of $\mathrm{CACH}$ at 56G. Another EST clone (343 bp) derived from mouse kidney (AA066584, referred to as mouse expressed sequence tag 2 in Table 2 and mEST2 in Fig. 3) was identical to the mouse CACH cDNA at nucleotide positions 1086-1428, suggesting the presence of a $\mathrm{CACH}$ transcript in mouse kidney. On the other hand, no sequence similarity was observed with other known proteins, demonstrating that $\mathrm{CACH}$ is a novel type of thioesterase.

Expression of recombinant $\mathrm{CACH}$ in $\mathrm{Sf9}$ insect cells. Infection of the Sf9 insect cells with the recombinant baculovirus containing the mouse full-length CACH cDNA (Fig. 1A) resulted in functional expression of recombinant $\mathrm{CACH}$ protein. The acetyl-CoA hydrolyzing activity was detected as early as two days after infection in the lysate prepared from infected cells, and was maximal at four days post-infection at each multiplicity of infection (MOI) 1, 5 and 10 (Fig. 4). The expressed enzyme activity was ATP-dependent and com-

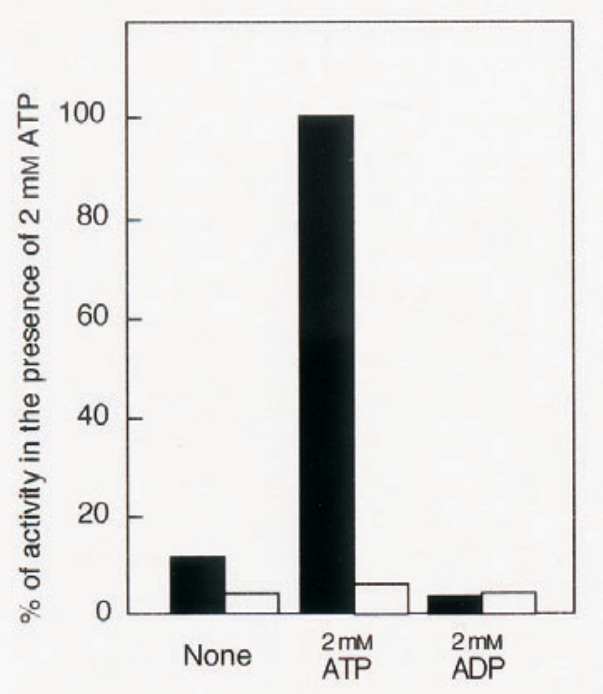

Figure 5. Effects of ATP and ADP on enzyme activity of mouse recombinant cytosolic acetyl-CoA hydrolase.

Acetyl-CoA hydrolase activity in cell lysates prepared from infected $(\square)$ or uninfected $(\square) S f 9$ cells was analyzed in the presence or absence of $2 \mathrm{mM}$ ATP or ADP. Values are shown as percentages of the activity in the presence of $2 \mathrm{mM}$ ATP. 
pletely inhibited by ADP (Fig. 5) like that of the rat $\mathrm{CACH}$. Furthermore, it exhibited cold lability (Fig. 6A) and its cold-inactivation could be partially abolished through incubation at $37^{\circ} \mathrm{C}$ in the presence of $0.16 \mathrm{mM}$ Triton $\mathrm{X}-100$ (Fig. 6B) like that of the rat recombinant as well as native enzyme.

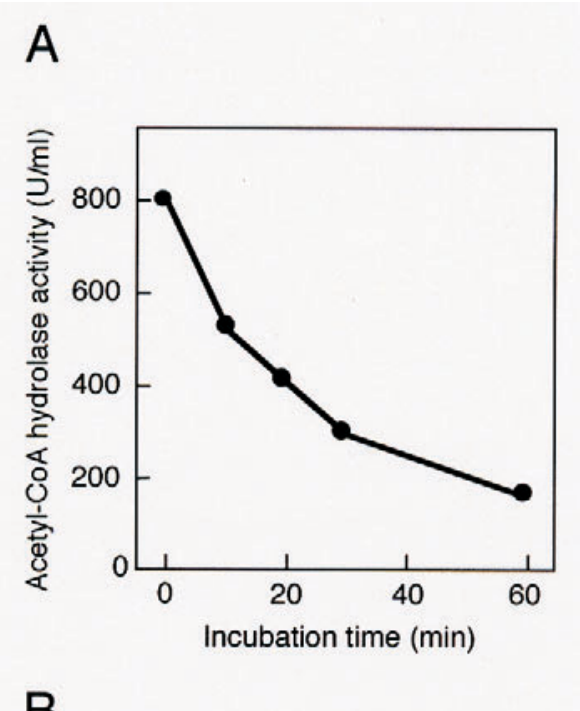

B

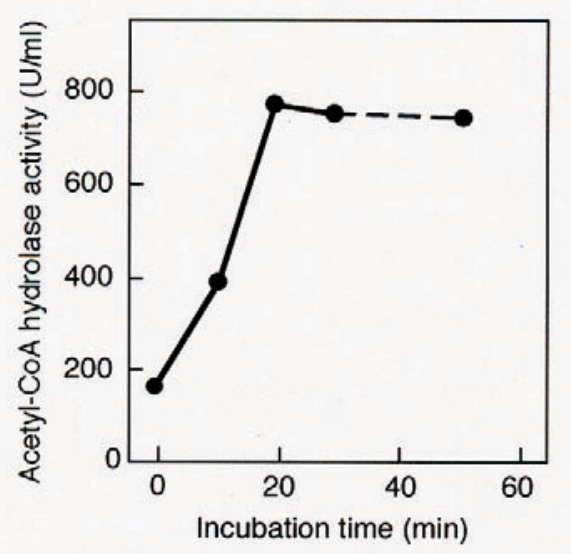

Figure 6. Time courses of cold inactivation (A) and reactivation (B) of mouse recombinant cytosolic acetyl-CoA hydrolase.

A. Cell-lysates prepared from infected $S f 9$ cells were incubated at $4^{\circ} \mathrm{C}$ for indicated times. Small aliquots were taken for measurement of remaining activity at $25^{\circ} \mathrm{C}$ in the presence of $2 \mathrm{mM}$ ATP. B. The cold inactivated sample was incubated at $37^{\circ} \mathrm{C}$ in the presence of $0.16 \mathrm{mM}$ Triton X-100 for $30 \mathrm{~min}$ (shown in full line) and then at $25^{\circ} \mathrm{C}$ for $20 \mathrm{~min}$ (shown in broken line). Small aliquots were taken at times indicated for measurement of remaining activity at $25^{\circ} \mathrm{C}$ in the presence of $2 \mathrm{mM}$ ATP.

\section{DISCUSSION}

A cytosolic acetyl-CoA hydrolase (CACH) is involved in fatty acid metabolism in rat liver (Matsunaga et al., 1985). The enzyme was induced by a single subcutaneous injection of a hypolipidemic drug about 6-fold in whole liver (Ebisuno et al., 1988). Marked increase of the enzyme activity was also observed in the acute but not in the chronic stage of streptozotocin-induced diabetic rats (Ebisuno et al., 1988). Insulin injection to the rats abolished this transient CACH induction (Ebisuno et al., 1988), suggesting that the enzyme contributes to adaptation to the abnormal lipid metabolic state (excessive increase in $\beta$-oxidation (Horie et al., 1986)), and a decrease in cholesterol biosynthesis (Lakshmanan et al., 1973).

In this study, the nucleotide sequence of mouse CACH cDNA was determined for the first time (Fig. 2). No targeting signals for organella were found in the sequence as well as in that of the rat homologue, suggesting cytoplasmic localization of the enzyme protein in rodent liver. This finding agrees well with our previous subcellular fractionation studies demonstrating that the rat liver acetyl-CoA hydrolase was exclusively located to the cytoplasm (Nakanishi et al., 1994). The deduced amino-acid sequence was $93.5 \%$ identical to that of the rat homologue (Table 2), and the encoded protein has a comparable calculated molecular mass to that of the rat enzyme (Suematsu et al., 2001). The authenticity of the cDNA was confirmed by its functional expression. As predicted from the over $90 \%$ identity of the mouse and rat $\mathrm{CACH}$ primary structures (Table 2), the recombinant mouse enzyme hydrolyzed acetyl-CoA in the presence of $2 \mathrm{mM}$ ATP just as the recombinant rat enzyme did. Besides the requirement for ATP as activator, the expressed recombinant mouse CACH protein was inactivated by cold, and was also reactivated in the presence of Triton X-100 at $37^{\circ} \mathrm{C}$ like the rat enzyme (Suematsu et al., 2001). Purification of the recombinant enzyme is required for further 
characterization, work is underway in our laboratory. Our experimental findings that the features of $\mathrm{CACH}$ were common to the two rodent species, together with bioinformatics data revealing that $\mathrm{CACH}$ was a novel type of thioesterase showing no sequence similarity with yeast acetyl-CoA hydrolase (Lee et al., 1990) and only poor similarity with cytosolic peroxisome proliferator-induced acyl-CoA thioesterase (Hunt et al., 1999; Broustas et al., 1996), suggest a conserved and specific role for this novel enzyme in the mammalian liver.

Two variants of mouse CACH cDNA were reported in the present paper. One had a 103-base pair deletion in the ORF, causing a frameshift mutation, resulting in a shorter ORF encoding only N-terminal 473 aminoacid residues (see Results). Whether it encodes an active enzyme remains to be examined. The other, encoding the normal $\mathrm{CACH}$ protein, was found to be a single-nucleotide polymorphism (AK004905, see Table 2). Further, two EST clones were also reported. One was a mouse liver EST clone (AI425375), suggesting another SNP of the $\mathrm{CACH}$ gene with a missense mutation. The other was a mouse kidney EST clone (AA066584), suggesting transcription of the $\mathrm{CACH}$ gene in mouse kidney. Further studies are required to determine genetic polymorphism and tissue- specific transcription of $\mathrm{CACH}$ affecting the catalytic activity of the enzyme.

In conclusion, this paper for the first time describes the entire cDNA sequence of mouse cytosolic acetyl-CoA hydrolase and its overproduction, allowing future genetic studies on the physiological functions and physicochemical characters of this novel enzyme. Phenotypic analyses of mouse models such as gene-destruction and targeted overexpression of $\mathrm{CACH}$ will provide useful approaches to conclusively establish the significance of the enzyme.

The authors would like to thank Junko Asano for her technical assistance.

\section{R E F E R E N C E S}

Berge RK, Aarsland A, Bakke OM, Farstad M. (1983) Hepatic enzymes, CoASH and long-chain acyl-CoA in subcellular fractions as affected by drugs inducing peroxisomes and smooth endoplasmic reticulum. Int $J$ Biochem.;15: 191-204.

Broustas CG, Larkins LK, Uhler MD, Hajra AK. (1996) Molecular cloning and expression of cDNA encoding rat brain cytosolic acyl-coenzyme A thioester hydrolase. J Biol Chem.; 271: 10470-6.

Ebisuno S, Isohashi F, Nakanishi Y, Sakamoto Y. (1988) Acetyl-CoA hydrolase: relation between activity and cholesterol metabolism in rat. Am J Physiol.; 255: R724-30.

Ebisuno S, Isohashi F, Nakanishi Y, Higashi T, Sakamoto Y. (1989) The biphasic change of cytosolic acetyl-CoA hydrolase in rat liver during 3'-methyl-4-dimethylaminoazobenzene hepatocarcinogenesis. Jpn J Cancer Res.; 80: $132-5$.

Horie S, Isobe M, Suga T. (1986) Changes in CoA pools in hepatic peroxisomes of the rat under various conditions. J Biochem.; 99: $1345-52$.

Hunt MC, Nousiainen SE, Huttunen MK, Orii KE, Svensson LT, Alexson EH. (1999) Peroxisome proliferator-induced long chain acyl-CoA thioesterases comprise a highly conserved novel multi-gene family involved in lipid metabolism. J Biol Chem.; 274: 34317-26.

Isohashi F, Nakanishi Y, Sakamoto Y. (1983a) Effects of nucleotides on a cold labile acetyl-CoA hydrolase from the supernatant fraction of rat liver. Biochemistry.; 22: 584-90.

Isohashi F, Nakanishi Y, Sakamoto Y. (1983b) Factors affecting the cold inactivation of an acetyl-coenzyme-A hydrolase purified from the supernatant fraction of rat liver. Eur $J$ Biochem.; 134: 447-52.

Isohashi F, Nakanishi Y, Matsunaga T, Sakamoto Y. (1984) A cold-labile acetyl-coenzyme-A hydrolase from the 
supernatant fraction of rat liver: reactivation and reconstitution of the active species from the inactive monomer. Eur J Biochem.; 142: 177-81.

Kozak M. (1987) At least six nucleotides preceding the AUG initiator codon enhance translation in mammalian cells. J Mol Biol.; 196: 947-50.

Kozak M. (1991) Structural features in eukaryotic mRNAs that modulate the initiation of translation. J Biol Chem.; 266: $19867-70$.

Lakshmanan MR, Nepokroeff CM, Ness GC, Dugan RE, Porter JW. (1973) Stimulation by insulin of rat liver $\beta$-hydroxy- $\beta$-methylglutaryl coenzyme A reductase and choresterol-synthesizing activities. Biochem Biophys Res Commun.; 50: 704-10.

Lee F-JS, Lin L-W, Smith JA. (1990) A glucose-repressible gene encodes acetyl-CoA hydrolase from Saccharomyces cerevisiae. $J$ Biol Chem.; 265: 7413-8.

Mannaerts GP, Debeer LJ, Thomas J, De Schepper PJ. (1979) Mitochondrial and peroxisomal fatty acid oxidation in liver homogenates and isolated hepatocytes from control and clofibrate-treated rats. $J$ Biol Chem.; 254: 4585-95.

Matsunaga T, Isohashi F, Nakanishi Y, Sakamoto Y. (1985) Physiological changes in the activities of extramitochondrial acetyl-CoA hydrolase in the liver of rats under various metabolic conditions. Eur $J$ Biochem.; 152: 331-6.

Nakanishi Y, Isohashi F, Ebisuno S, Sakamoto Y. (1988) Binding of nucleotides to an extramitochondrial acetyl-CoA hydrolase from rat liver. Biochemistry.; 27: 4822-6.

Nakanishi Y, Okamoto K, Isohashi F. (1993) Effects of chronic administration of the peroxisome proliferator, clofibrate, on cytosolic acetyl-CoA hydrolase in rat liver Biochem Pharmacol.; 45: 1403-7.

Nakanishi Y, Okamoto K, Isohashi F. (1994) Subcellular distribution of ATP-stimulated and ADP-inhibited acetyl-CoA hydrolase in livers from control and clofibrate-treated rats: comparison of the cytosolic and peroxisomal enzyme. J Biochem.; 115: $328-32$.

Prass RL, Isohashi F, Utter MF. (1980) Purification and characterization of an extramitochondrial acetyl coenzyme A hydrolase from rat liver. $J$ Biol Chem.; 255: $5215-23$.

Simon EJ, Shemin D. (1953) The preparation of S-succinyl coenzyme A. J Am Chem Soc.; 75: 2520 .

Söling H-D, Rescher C. (1985) On the regulation of cold-labile cytosolic and of mitochondrial acetyl-CoA hydrolase in rat liver. Eur $J$ Biochem.; 147: 111-7.

Suematsu N, Okamoto K, Isohashi F. (1996) Effects of various proteins or peptides on reactivation of cold-inactivated acetyl-CoA hydrolase from rat liver. St Marianna Med J.; 24: $691-7$.

Suematsu N, Okamoto K, Shibata K, Nakanishi Y, Isohashi F. (2001) Molecular cloning and functional expression of rat liver cytosolic acetyl-CoA hydrolase. Eur J Biochem.; 268: 2700-9. 